\title{
"SI RIMOVERÀ ADUNQUE LA NOSTRA FANCIULLA IN TUTTO DALL'USO DELLA MUSICA", MUSICA E STRUMENTI MUSICALI NELL'EDUCAZIONE FEMMINILE DEI PAESI BASSI NEL XVI SECOLO
} "Si rimoverà adunque la nostra fanciulla in tutto dall’uso della musica», music and musical
instruments in female education in the Netherlands in the 16th century"

“«Si rimoverà adunque la nostra fanciulla in tutto dall’uso della musica», música e instrumentos musicales en la educación femenina de los Países Bajos en el siglo XVI"

\section{Maddalena Bellavitis ${ }^{1}$}

Recibido: 30-06-2020

Aceptado: $10-08-2020$

\section{Resumen}

Este artículo pretende analizar algunas iconografías femeninas relacionadas con la música a la luz de los tratados y teorizaciones que en el Renacimiento consideraron la relación entre las jóvenes mujeres de una buena familia, los instrumentos musicales y el canto. Inicialmente se citan algunos manuales para guiar la educación de las jóvenes, luego se consideran algunos grupos de pinturas que representan a mujeres tocando: principalmente ejemplos de María Magdalena tocando el laúd o el clavicordio, alegorías o retratos.

Palabras clave: Renacimiento, música, educación femenina, Países Bajos, Maestro de las Medias Figuras, Jan van Hemessen, María Magdalena.

\section{Abstract}

This article intends to analyze some female iconographies related to music in view of the treatises and theorizations that in the Renaissance considered the relationship between upper class girls, musical instruments and singing.

Initially the article mentions some manuals written to guide the education and training of young girls, and after that it will consider some groups of paintings depicting young ladies playing: mainly examples of Mary Magdalene playing, allegories or portraits.

Keywords: Renaissance, music, female education, Netherlands, Master of the Female Half-Lengths, Jan van Hemessen, Mary Magdalene.

1 Università degli Studi di Udine, maddalena.bellavitis@gmail.com 
Sulle donne e la musica nel Rinascimento si è scritto e si scrive molto. Si trovano numerosi studi che analizzano gli usi e i contesti in cui a servirsi di compositori e musicisti sono state committenze femminili, ma anche i casi in cui, per diletto o professione, erano le dame stesse a incaricarsi dell'esecuzione musicale ${ }^{2}$. La maggior parte della bibliografia si è occupata delle corti italiane, principalmente quelle di Ferrara e Mantova, e delle figure che per rapporti di parentela o per ingaggi professionali venivano in contatto con i loro rappresentanti. Se Isabella d'Este è la protagonista della maggior parte delle ricerche svolte, grande attenzione è stata dedicata anche a Beatrice d'Aragona, Lucrezia Borgia, o ad un'artista come Anna inglese ${ }^{3}$.

Per quanto riguarda il fare musica, l'ambito italiano è stato un soggetto di studio particolarmente soddisfacente anche per l'abbondanza di documentazione e per gli importantissimi apporti offerti dai contemporanei in merito al comportamento e all'educazione femminile ${ }^{4}$ Grazie a contributi come la fondamentale opera di Baldesar Castiglione, possiamo sapere se e come una dama poteva cantare o suonare in pubblico, e tutte le possibili opzioni e sfumature che ne regolavano l'approccio a strumenti e compagnie musicali ${ }^{5}$ :

2 Per citare alcune opere a carattere generale: BORROFF, E. Women Composers: Reminiscence and History. College Music Symposium. 1975, vol. 15, p. 26-33; BOWERS, J. M., TICK, J., editori. Women Making Music: The Western Art Tradition, 1150-1950. Urbana e Chicago: University of Illinois Press, 1987; PRIZER, W. F. Games of Venus: Secular Vocal Music in the Late Quattrocento and Early Cinquecento. The Journal of Musicology, vol. 9, $\mathrm{n}^{\circ}$ 1, 1991, p. 3-56; LORENZETTI, S. "Quel celeste cantar che mi disfa$c e$ ". Immagini della donna ed educazione alla musica nell'ideale pedagogico del Rinascimento italiano. Studi Musicali, 1994, Anno XXIII, n 2, p. 241-262; STEBLIN, R. The Gender Stereotyping of Musical Instruments in the Western Tradition. Canadian University Music Review / Revue de musique des universités canadiennes, Voices of Women: Essays in Honour of Violet Archer Voix de femmes. Mélanges offerts à Violet Archer, 1995, vol. 16, n 1, p. 128-144; BRYCE, J. Performing for Strangers: Women, Dance, and Music in Quattrocento Florence. Renaissance Quarterly, 2001, vol. 54, n 4, Part 1, p. 1074-1107; DENNIS, F. Unlocking the Gates of Chastity Music and the Erotic in the Domestic Sphere in Fifteenth- and Sixteenth-Century Italy. In: S. F. MATTHEWS-GRIECO, editore. Erotic cultures of Renaissance Italy. Visual Culture in Early Modernity. Aldershot: Ashgate, 2010, p. 223-246; COLTON, L., HAWORTH, C., editori. Gender, Age and Musical Creativity. Farnham: Ashgate Publishing, 2015; LAMARY, T. Musical Voices of Early Modern Women: Many-Headed Melodies. Londra: Routledge, 2017.

3 Si vedano ad esempio: PRIZER, W. F. Isabella d'Este and Lorenzo Da Pavia, 'Master Instrument-Maker'. Early Music History, 1982, n 2, p. 87-127; PRIZER, W. F. Isabella d'Este and Lucrezia Borgia as Patrons of Music: The Frottola at Mantua and Ferrara. Journal of the American Musicological Society, 1985, vol. 38, n 1, p. 1-33; PRIZER, W. F. Una "Virtù Molto Conveniente A Madonne": Isabella D'este as a Musician. The Journal of Musicology, 1999, vol. 17, n 1, A Birthday Tableau for H. Colin Slim, p. 10-49; LORENZETTI, S. Public behavior, music and the construction of feminine identity in the Italian Renaissance. Recercare, 2011, vol. 23, n 1-2, p. 7-34; SHEPHARD, T. Constructing Isabella d'Este's musical decorum in the visual sphere, Renaissance Studies, 2011, vol. 25, n 5, p. 684-706; BLACKBURN, B. J., Anna Inglese and Other Women Singers in the Fifteenth Century: Gleanings from the Sforza Archives. In: Kristine Forney e Jeremy L. Smith, editori. Sleuthing the Muse: Essays in Honor of William F. Prizer,Festschrift Series n²6. Hillsdale, NY:Pendragon Press, 2012, p. 237-52; PRIZER, W. F. Renaissance Women as Patrons of Music: The North-Italian Courts. In: Thomas. SCHMIDT-BESTE, editore. Institutions and Patronage in Renaissance Music. Aldershot: Ashgate, 2012, p. 161-190; SHEPHARD, T. Echoing Helicon: Music, Art and Identity in the Este Studioli, 1440-1530. Oxford: Oxford University Press, 2014; STRAS, L. Women and Music in Sixteenth-Century Ferrara (New Perspectives in Music History and Criticism).Cambridge: Cambridge University Press, 2018; COMELLAS SOLÉ, J. La música dentro de las cortes europeas del siglo XVI. El modelo de Il Cortegiano y el papel de las damas en su consolidación. El ejemplo de las cortes italianas. Philostrato. Revista de Historia y Arte. 2020, n 7,p. 5-32 [consultato: 3 luglio 2020]. ISSN 2530-9420. Disponibile alla pagina: https://doi.org/10.25293/philostrato.2020.01.

4 JARDINE, L. 'O Decus Italiae Virgo', or The Myth of the Learned Lady in the Renaissance. The Historical Journal, 1985 , vol. 28, n 4, p. 799-819; SCHIESARI, J. In Praise of Virtuous Women? For a Genealogy of Gender Morals in Renaissance Italy. Annali d'Italianistica, 1989, vol. 7, Women's Voices in Italian Literature, p. 66-87; LORENZETTI, S. "Quel celeste cantar che mi disface". Immagini della donna ed educazione alla musica nell'ideale pedagogico del Rinascimento italiano. Studi Musicali, 1994, Anno XXIII, $\mathrm{n}^{\circ}$ 2, p. 241-262; CAGNOLATI, A. Giovanni Michele Bruto e l'educazione femminile: La Institutione di una fanciulla nata nobilmente (1555). Annali dell'Univeristà di Ferrara, Nuova serie, Filosofia, Discussion Papers. 2001, $\mathrm{n}^{\circ}$ 64, p. 1-22; VECCE, C. Modelli della pedagogia umanistica dall 'Italia all'Europa. In: Maria Da Rif, editore. Civiltà italiana e geografie d'Europa. XIX Congresso AISLLI 19-24 settembre 2006, Trieste - Capodistria - Padova - Pola. Trieste: EUT Edizioni Università di Trieste, 2009, p. 146-154.

5 CASTIGLIONE, B. Il cortegiano. Venezia: Eredi di Aldo Manuzio, 1545; SCHNEIDER, F. An Amused Muse: The donna di palazzo and Music in the Cortegiano, L'analisi linguistica e letteraria, 2002, vol. 10, n 1-2, p. 449-460; COMELLAS SOLÉ, J. La música dentro de las cortes europeas del siglo XVI. El modelo de Il Cortegiano y el papel de las damas en su consolidación. El ejemplo de las 
Poich'io poso formar questa Donna a modo mio, non solamente non uoglio ch'ella usi questi esercitii virili cosi robusti, $\&$ asperi, ma voglio che quegli anchora, che son convenienti a donna, faccia con riguardo, \& con quella molle delicatura, che havemo detto convenirsele:\& però nel danzar non vorrei vederla usar movimenti troppo gagliardi, \& sforzati: ne meno nel cantar o sonar quelle diminutioni forti,\& replicate che mostrano più arte che dolcezza: medesimamente gli instrumenti di Musica, che ella usa (secondo me) debbono esser conformi a questa intentione imaginatevi come disgratiata cosa saria veder una donna sonare tmbburri, piffario trombe, o altri tali instrumenti: \& questo perché la loro asprezza nasconde, e leva quella soaue mansuetudine, che tanto adorna ogni atto,che faccia la donna: però, quando ella viene a danzar, o fare musica di che sorte si sia, deve indurvisi con lasciarfene alquanto pregare: \& con una certa timidità, che mostri quella nobile vergogna, che é contraria della impudentia.

La critica non ha naturalmente tralasciato le altre geografiche, come ad esempio i Paesi Bassi. In questo caso gli ovvi catalizzatori di interesse erano Margherita d'Asburgo e Maria d'Ungheria: le due nobildonne, affascinanti punti di riferimento per la cultura e il gusto del loro tempo, sono tra le principali fonti di ispirazione degli studiosi per il loro amore per la musica e per la loro vasta competenza in materia ${ }^{6}$. Grande interesse è stato anche rivolto a trattati e manuali, la cultura neerlandese, come quella italiana, ha visto un profondo impegno nella teorizzazione dell'educazione delle giovani fanciulle, e tra i vari aspetti dibattuti la componente musicale non veniva certo trascurata. La trattatistica italiana era nota e spesso presa come punto di riferimento, sia per gli stretti contatti con l'Italia mantenuti e intensificati anche da quanti dalla Penisola si erano trasferiti nelle Fiandre, sia per la familiarità dei locali con numerose lingue che rendevano accessibili al pubblico neerlandese anche opere non ancora tradotte in fiammingo, come il Cortegiano, che nonostante non fosse stato pubblicato nei Paesi Bassi in una versione vernacola prima della seconda metà del XVII secolo ${ }^{7}$, poteva tranquillamente essere letto nella stesura originale o nelle sue traduzioni francesi. Il rapporto tra le donne e la musica in Fiandra non è stato studiato solo attraverso i documenti e le pubblicazioni del tempo $^{8}$, le fonti visive hanno infatti offerto a musicologi e storici dell'arte gli spunti per riflessioni e considerazioni sui gusti musicali e sulle modalità di esecuzione del tempo. Tra le opere in questione rientrano alcuni gruppi di dipinti - come scene di genere, figure di sante e ritratti di famiglia - e certe stampe, principalmente di soggetto allegorico. La maggior parte di essi sono ben noti alla critica, ma

cortes italianas. Philostrato. Revista de Historia y Arte. 2020, n 7,p. 5-32 [consultato: 3 luglio 2020]. ISSN 2530-9420. Disponibile alla pagina: https://doi.org/10.25293/philostrato.2020.01.

6 Si vedano ad esempio: HARASZTI, E. Marie de Hongrie et son Ungarescha. Revue de Musicologie, 1930, vol. 11, n 35, p. 176194; PICKER, M. The Chanson Albums of Marguerite of Austria: Manuscripts 228 and 11239 of the Bibliothèque Royale de Belgique, Bruxelles. Berkeley: University of California Press, 1960; DUGGAN, M. K. Queen Joanna and Her Musicians. Musica Disciplina, 1976, vol. 30, p. 73-95; GOSS THOMPSON, G. Mary of Hungary and Music Patronage. The Sixteenth Century Journal, $1984 a$, vol. 15, $\mathrm{n}^{\circ}$ 4, p. 401-418; GOSS THOMPSON, G. Music in the Court Records of Mary of Hungary, Tijdschrift van de Vereniging voor Nederlandse Muziekgeschiedenis, 1984b, vol. 34, n² 2, p. 132-173; Moens-Haenen, G. Music at the court of Marguerite of Austria. Jaarboek van het Vlaams Centrum voor Oude Muziek. 1987; MECONI, H. Pierre de la Rue and Musical Life at the Habsburg-Burgundian Court. Oxford: Oxford University Press, 2003.

7 FRIJHOFF, W., e SPIES, M. Dutch Culture in a European Perspective: 1650, Hard-Won Unity. Assen: Van Gorcum, 2004, p. 571.

8 FORNEY, K. K. Nymphes gayes en abry du Laurier: Music Instruction for the Bourgeois Woman. Musica Disciplina, 1995, vol. 49, p. 151-187; FORNEY, K. K. A Proper Musical Education for Antwerps Women. In: Susan FORSCHER WEISS, R. Eugene MURRAY Jr., Cynthia. J. CYRUS, editori. Music Education in the Middle Ages and the Renaissance Indiana. Bloomington: Indiana University Press, 2010, p. 84-125, con bibliografia precedente. 
sono stati studiati principalmente considerando uno specifico aspetto, stilistico, iconografico o musicale, tralasciando spesso un tentativo di visione d'insieme che li riconducesse ad una sensibilità più generale e al contesto peculiare della cultura neerlandese dell'epoca. Può quindi essere utile rivederne le caratteristiche principali alla luce di tutte le componenti e le caratteristiche già emerse, e in rapporto con la contemporanea letteratura dedicata all'educazione e alla formazione femminili.

Per prima cosa si rivedranno le posizioni dei trattatisti del tempo nei confronti della musica, e quali si ritenevano essere i comportamenti e le abitudini che una giovane dovesse assumere nei confronti del canto e dell'esecuzione strumentale. Nel XVI secolo la musica era considerata una componente fondamentale dell'educazione, e a conferma di quanto ci raccontano manuali e documenti del tempo, il fatto che suonare e cantare fossero una tradizione comune alla popolazione è confermata dalla $D e$ scrittione di Guicciardini, in cui si legge che

l'hanno [la musica] tanto propria \& naturale, che huomini, \& donne cantan naturalmente a mifura, con grandifsima gratia, \& melodia, onde hauendo poi congiunta l'arte alla natura, fanno \& divoce, \& di tutti gli ftrumenti quella pruoua, \& harmonia, che fi vede $\&$ ode, talche fe ne truoua fempre per tutte le Corti de Principi Chriftiani9.

Kristine Forney, nei suoi numerosi interventi sulla situazione musicale nei Paesi Bassi, ha pubblicato alcuni contratti che testimoniano la cura che le famiglie avevano nel far imparare la musica alle proprie figlie. Si tratta di documenti relativi all'ingaggio di maestri privati per insegnare alle figlie di facoltosi mercanti non solo le materie fondamentali per un'istruzione di base, ma anche a suonare, e alcuni di essi riportano le spese sostenute per lezioni private di canto delle allieve della Scuola dell'Albero di Alloro (De Lauwerboom $)^{10}$. Oltre alla capacità di cantare e suonare uno o più strumenti, alcune ragazze acquisivano inoltre le conoscenze necessarie per comporre o quantomeno trascrivere musica, come si può vedere in un ritratto recentemente proposto dall'antiquario olandese Kollenburg $^{11}$ (Fig. 1). Il dipinto, che chi scrive ritiene molto vicino allo stile e alle tematiche di Jan van Hemessen, mostra una giovane che si appoggia ad un clavicordo mentre traccia delle note su un foglio.

Va inoltre ricordato che Anversa, grazie all'attività editoriale che vi fioriva, ha visto la pubblicazione di numerosi testi legati all'educazione, spesso manuali impiegati nelle scuole, - tra cui la Scuola dell'Albero di Alloro - come supporto all'istruzione delle proprie alunne. Vi si fornivano esempi di buona condotta e morale cristiana, e si proponevano situazioni esemplari di vita quotidiana, spesso in più lingue per favorire l'apprendimento o il perfezionamento anche di altri idiomi. Nella Guirlande

9 GUICCIARDINI, L. Descrittione Di M. Lodovico Gvicciardini patritio fiorentino, di tutti i Paesi Bassi, altrimenti detti Germania Inferiore: Con più carte di Geographia del paese, \& col ritratto naturale di più terre principali. Anversa: Guglielmo Silvio, 1567, p. 28.

10 FORNEY, K. K. A Proper Musical Education for Antwerps Women. In: Susan FORSCHER WEISS, Russell E. MURRAY Jr., Cynthia J. CYRUS, editori. Music Education in the Middle Ages and the Renaissance Indiana. Bloomington: Indiana University Press, 2010, p. 84-125, p. 88 e p. 103.

11 Olio su tavola, cm 26.4 x 21.2 (come Maestro delle Mezze Figure). Un sentito ringraziamento va a Mischo van Kollenburg per la sua collaborazione e per la gentilezza con cui ha fornito e permesso la pubblicazione di una riproduzione del dipinto, e ringrazio anche il Prof. Massimo Ossi per i preziosi commenti su quanto la fanciulla sta scrivendo. 


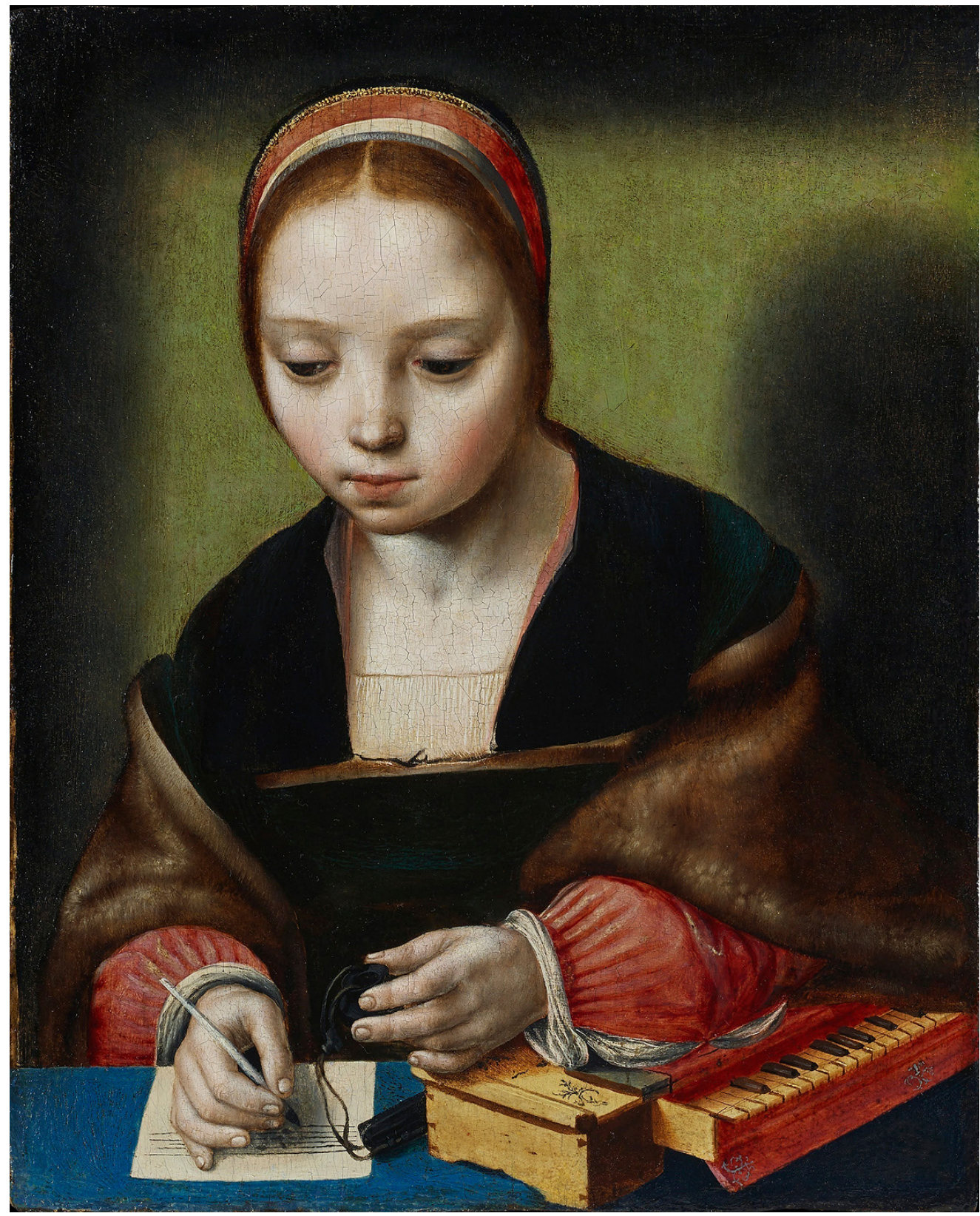

Fig. I: Maestro delle Mezze Figure Femminili, Fanciulla al verginale, mercato (su gentile concessione di Kollenburg Antiquairs, Oirschot) (C) Kollenburg Antiquairs.

des jeunes filles, pubblicata per la prima volta nel 1564, Gabriel Meurier inseriva un dialogo in cui alcune fanciulle si dedicavano a vari passatempi, tra cui la musica ${ }^{12}$ : una, Corneille, proponeva di suonare la spinetta, mentre un'altra, Lucie, si accertava che le altre sapessero suonarla. Ancora più interessante è come una terza protagonista del dialogo, Alison, dichiarasse che lo strumento fosse scordato e desse indicazioni per accordarlo, dimostrando come le conoscenze musicali femminili non si limitassero alla pratica ma comprendessero anche la sistemazione degli strumenti. Le protagoniste del dialogo della Guirlande des jeunes filles decidevano poi di cantare una canzone, e Lucie propendeva per una canzone devota (“Chantons quelque cantique spirituel'), confermando l'avversione per la musica lasciva che era stata bandita dagli intrattenimenti per le fanciulle per bene già da Quintiliano. Anche un'istitutrice, Magdaleine Valéry, ammoniva la sua allieva Emerence contro il

12 Si veda l'edizione rivista e ampliata della traduzione tedesca: MAURIER, G. La guirlande des Jeunes filles, bastie et composee en langue francoise et flamengue, et de nouveau reveue et translatee de francois en haut alleman par Abraham des Mans. Edizione: Colonia, 1597, p. 99. 
suonare danze, considerandole troppo mondane ${ }^{13}$.Questo era anche il pensiero delle principali e più autorevoli autorità in materia, Luis Vives ed Erasmo da Rotterdam. Luis Vives, nel suo De institutione feminae Christianae, aveva scritto che una donna non dovrebbe mostrarsi in pubblico mentre suona o canta, e avvertiva che se proprio voleva cantare doveva farlo "doulcement" e che avrebbe dovuto conoscere solo "chansons honnestes, graves \& decentes" 14 , mentre Erasmo tra le pagine della sua Christiani matrimonii institutio condannava fermamente i genitori che lasciavano che le proprie figlie cantassero canzoni mondane, i soggetti di tali canzoni, e anche i loro autori, accusandoli aspramente di corrompere la gioventù:

Iam apud quasdam nationes etiam solenne est quotannis ædere novas cantiones, quas ediscunt puellæ. Quarum argumentum fere tale est: Delufus ab uxore maritus; aut puella frustra servata a parentibus, aut furtivus cum amatore concubitus. Atque haecita referuntur quasi bene gesta, applauditurque felicinequitiae. Pestilentibus argumentis additur tanta sermonis obscoenitas, per metaphoras \& allegorias, utipsa turpitudo nonpossit loqui turpius. Ethic quæstus alit multos, præsertimapud Flandros. Si leges vigilarent, talium næniarum artifices deberent cæsi flagris sub carnifice, pro lascivis lugubres canere cantilenas. Et hi qui publice corrumpunt iuventutem, ex scelere vivunt. Et inveniuntur parentes, qui putent esse civilitatis partem, filiam non ignorare talia carmina... ${ }^{15}$.

Il canto presentava dunque una situazione complessa, essendo oggetto di alcune posizioni fortemente contraddittorie. Johannes Tinctoris, forse anche per l'influenza dell'ambiente italiano, nel suo Complexus effectuum musices aveva mostrato un atteggiamento molto aperto, citando Ovidio e incitando le fanciulle a sfruttare la grazia del canto. Il compositore suggeriva addirittura di usarlo per conquistare l'amore: "Unde Ovidius puellis amorem virorum allicere cupientibus praecipit ut cantare discant. Enimvero in tertio libro De arte amandi sic inquit: 'Res est blanda canor: discant cantare puellae"'16. Senza arrivare a questi estremi, secondo la maggior parte delle posizioni alle fanciulle di buona famiglia era permesso cantare, purché si trattasse di salmi, testi liturgici, o canzoni di tematica devozionale e morale. Tra i testi redatti nel corso del XVI secolo ce ne sono tuttavia alcuni che, rifacendosi alla posizione di San Girolamo (Lettera CVII), rifiutavano ogni qualsiasi coinvolgimento femminile con la musica ${ }^{17}$. Tra questi va citato Giovanni Michele Bruto, che durante il suo soggiorno anversese aveva pubblicato La institutione di una fanciulla nata nobilmente, in italiano e in francese. Rivolgendosi alla giovanissima Marietta Cattaneo, Bruto la metteva in guardia contro le insidie della musica ("tuttavia sotto nome di virtù ... porta ella dolce \& soave esca à gravi \& importanti mali" e "poiche ella

13 VALERY, M. La Montaigne des pucelles. Den maeghdenbergh. Leiden: Jan Paets, 1599.

14 MAURIER, G. La guirlande des Jeunes filles, bastie et composee en langue francoise et flamengue, et de nouveau reveue et translatee de francois en haut alleman par Abraham des Mans. Edizione: Colonia, 1597, p. 76.

15 ERASMO, D. Christiani matrimonii institutio, Apud Inclytam Basileam, 1526.

16 Si veda: TINCTORIS, J. Complexus effectuum musices, $1472-75$ [consultato: 26 giugno 2020]. Disponibile alla pagina http://www. chmtl.indiana.edu/tml/15th/TINCOM1 TEXT.html, capitolo XVII.

17 Si deve però notare che nel caso della lettera a Pacatula, Girolamo in realtà invitava a far cantare i salmi alla piccola promettendole monetine come premio (Lettera CXXVIII), e va specificato che il santo si riferiva ad una bambina destinata alla vita monastica, e quindi ad una situazione differente da quella delle future dame neerlandesi. 
apre l'adito a' molti vitij, sotto honesto velo di virtù'), del canto (facendo l'esempio delle sirene), e contro il rischio di essere giudicata in maniera negativa ${ }^{18}$.

Venendo quindi agli esempi che ci sono stati lasciati dagli artisti, il gruppo più noto e dibattuto ripropone una composizione associata al nome del Maestro delle Mezze Figure che viene conservata in almeno quattro versioni (Fig. 2). Tre bellissime musiciste eseguono "Jouyssance vous donneray", una canzone composta da Claudin de Sermisy con testo di Clément Marot, pubblicata per la prima volta nel $1535^{19}$. Una delle tre donne canta, le altre due suonano il liuto e il flauto traverso. L'intervento più significativo sui quattro dipinti è dovuto a Colin Slim, che ha analizzato attentamente la musica suonata dalla flautista, il foglio seguito dalla cantante e

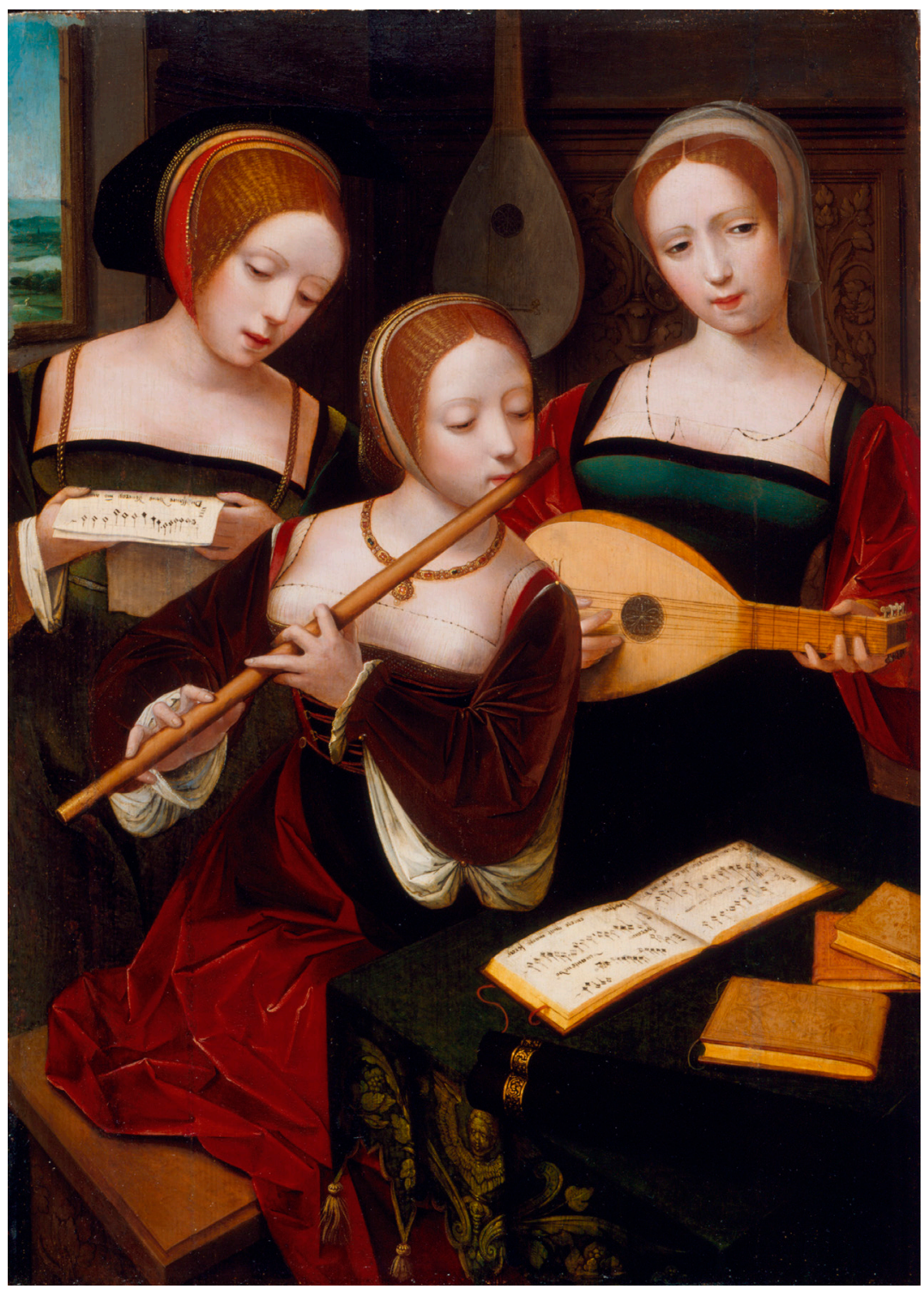

Fig. 2: Maestro delle Mezze Figure, Concerto, Los Angeles County Museum of Art (C) Los Angeles County Museum of Art.

l'esecuzione strumentale di flauto e liuto ${ }^{20}$. Nonostante la bocca chiusa della cantante e l'apparenza angelica e lo sguardo abbassato di tutte e tre - caratteristiche che, assieme al fatto che siano da sole, rimandano senz'altro alla modestia imprescindibile per una donna di natali elevati di quell'epoca -la critica ha osservato che i dettagli e le simbologie che caratterizzano la composizione alludono a tutt'altro contesto. Innanzi tutto, invece che un salmo o una canzone adatta ad un orecchio verginale, le tre musiciste si cimentano in una canzone d'amore, ossia quanto di più proibito si potesse menzionare nei testi formativi (si è appena ribadito come anche le posizioni più accondiscendenti fossero assolutamente contrarie alle canzoni d'amore). A peggiorare lo scenario si aggiunge la scelta degli strumenti: il flauto e il liuto. L'allusività erotica che entrambi comportavano è stata ribadita in vari

18 BRUTO, G. M. La institutione di una fanciulla nata nobilmente. Anversa: Jehan Bellére, 1555, pp. 36-38.

19 ATTAINGNANT, P. Premier livre contenant XXXI chansons musicales, Paris, 1535.

20 PARKINSON, J. A. A Chanson by Claudin de Sermisy. Music \& Letters, 1958, vol. 39, № 2, p. 118-122, ma soprattutto COLIN SLIM, H.. Paintings of Lady Concerts and the Transmission of "Jouissance vous donneray". Imago Musicae, 1984, n I, p. 51-73, con bibliografia precedente. 
interventi sull'iconografia musicale del rinascimento, venivano generalmente associati alle cortigiane e alle donne dai costumi discutibili. De Mirimonde, in riferimento ad un'altra serie di dipinti a tema musicale, un gruppo di Concerti campestri spesso associati alla parabola del figliol prodigo, scriveva come i due strumenti insieme formassero una "coppia" (tra virgolette nel testo) ben equilibrata ${ }^{21}$. Friedländer ${ }^{22}$ aveva notato che un riferimento al liuto in questo senso e collegato ai quadri fiamminghi che raffiguravano donne che suonano il flauto tedesco (flauto traverso) era presente in una fonte di poco successiva al periodo di esecuzione di questi dipinti, Les Vies des dames galantes di Brantôme ${ }^{23}$, ma il flauto era uno strumento proibito alle dame anche per una considerazione estetica, basti pensare al già citato brano in cui Castiglione metteva in guardia le donne contro la poca grazia che si dimostrava suonando uno strumento a fiato ${ }^{24}$. I riferimenti sessuali relativi al liuto all'epoca erano dunque tranquillamente sbandierati ${ }^{25}$ - il nome stesso dello strumento si prestava a giochi di parole - e lo strumento era protagonista di dipinti e poemi.

Proprio il liuto accompagnava un'altra serie di dipinti che illustravano figure femminili che suonano, un altro gruppo legato all'attivissima bottega del Maestro delle Mezze Figure e che ha come protagonista Maria Maddalena. Le versioni in questo caso sono molte, si differenziano per alcuni dettagli e per la diversa qualità stilistica. Come i Concerti appena citati, anche queste Maddalene sono state oggetto di studi approfonditi da parte dei musicologi, che ne hanno analizzato i dettagli esecutivi e ne hanno spesso identificato i testi suonati ${ }^{26}$. A loro volta bellissime, queste Maddalene suonano il liuto seguendo la partitura di quelle che sono state ancora una volta identificate con canzoni d'amore, Jouyssance vous donneray inclusa. Si tratta quindi, come nel caso delle loro colleghe dei Concerti, di nuovo di trasgressioni rispetto a quello che all'epoca era considerato il decoro delle donne oneste. Nonostante praticamente in tutte le versioni la santa tenga gli occhi bassi sullo spartito, è innegabile come i riferimenti ad una situazione non esemplare fossero ovvi. A questi si aggiungevano le vicende biografiche di Maria Maddalena e tutta la vastissima produzione letteraria che l'aveva vista protagonista, descrivendola già dal medioevo come una peccatrice frivola e dedita solo al divertimento ${ }^{27}$. Vale la pena soffermarsi soprattutto su commedie e sacre rappresentazioni, come la Mondanité de

21 DE MIRIMONDE, A. P. La musique chez les peintres de la fin de l'ancienne école de Brouges. Jaarboek van het Koninklijk Museum voor Schone Kunsten - Antwerpen. 1976, p. 25-81, p. 63.

22 FRIEDLÄNDER, M. J. Early Netherlandish Paintings. XII. Jan van Scorel and Pieter Coeck van Aelst. Edizione a cura di Henri PAUWELS e Geert LEMMENS. Leida: Sijthoff, e Bruxelles: La Connaissance, 1975, p. 19.

23 DE BOURDEILLE DE BRANTOME, P. Vies des dames galantes. Edizione Frères Garnier, Parigi, 1841, p. 188.

24 All'epoca dell'esecuzione di questi dipinti una traduzione fiamminga del Cortegiano non esisteva ancora, ma le edizioni italiane e francesi erano perfettamente accessibili al pubblico neerlandese che parlava entrambe le lingue senza difficoltà (si veda nota 7).

25 Per il liuto e la sua simbologia in arte e letteratura si veda: ZECHER, C. The Gendering of the Lute in Sixteenth-Century French Love Poetry. Renaissance Quarterly, 2000, vol. 53, n³, p. 769-791.

26 Si vedano di nuovo PARKINSON, J. A. A Chanson by Claudin de Sermisy. Music \& Letters, 1958, vol. 39, N 2, p. 118-122, e COLIN SLIM, H. Paintings of Lady Concerts and the Transmission of "Jouissance vous donneray". Imago Musicae, 1984, n' I, p. 5173, ma anche COLIN SLIM, H. Mary Magdalene, Musician and Dancer. Early Music, 1980, Vol. 8, n 4, p. 460-473. ISSN 0306-1078, e HEARTZ, D. Mary Magdalen, Lutenist. Journal of the Lute Society of America, 1972, n 5, p. 52-67.

27 Si vedano: BELLAVITIS, M. Tra ritratto e devozione, immagini della Maddalena nella prima metà del Cinquecento, Critica d'Arte.2013 [2015], LXXV, n 55-56, p. 19-36, con bibliografia relativa, e VAN PELT, N. T. Drama in Medieval and Early Modern Europe: Playmakers and their Strategies. Londra: Routledge, 2019, pp. 39-64. 


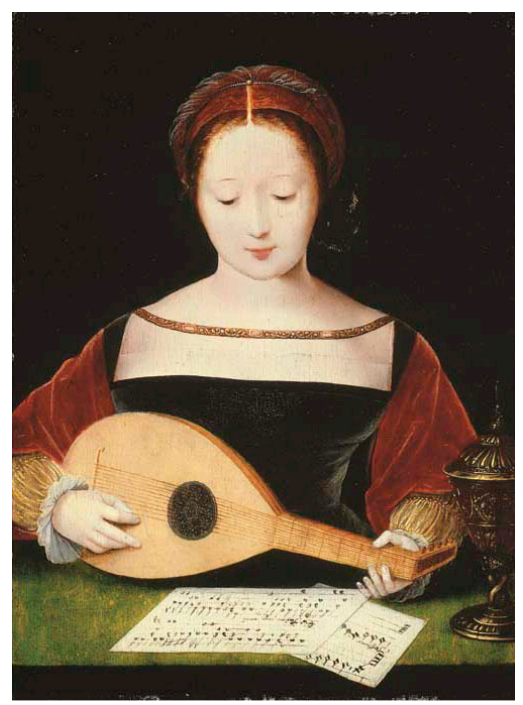

Fig. 3: Maestro delle Mezze Figure, Maria Maddalena che suona il liuto, attuale collocazione sconosciuta.

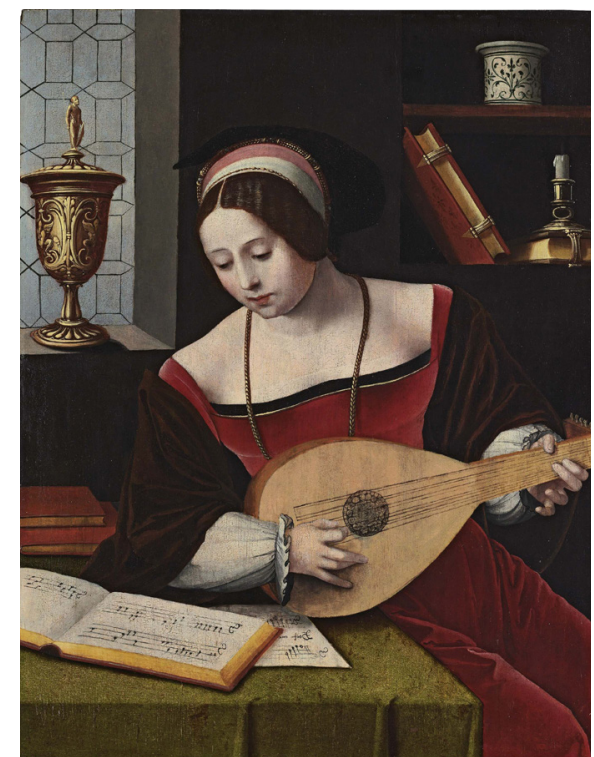

Fig. 4: Maestro delle Mezze Figure, Maria Maddalena che suona il liuto, attuale collocazione sconosciuta.

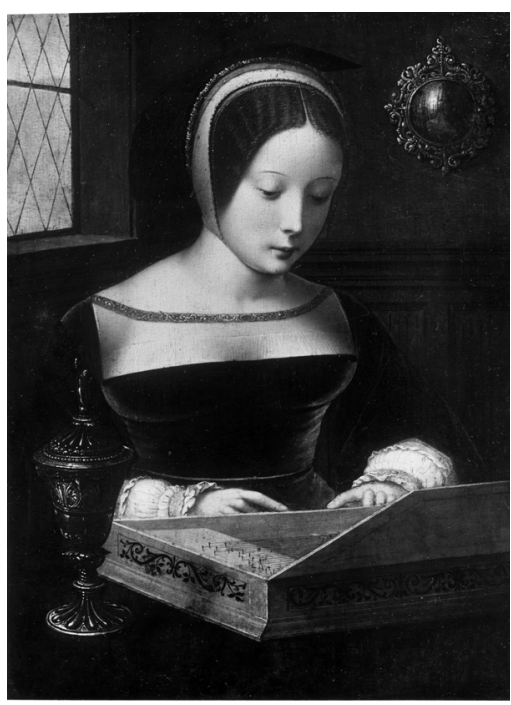

Fig. 5: Maestro delle Mezze Figure, Maria Maddalena che suona il clavicordio, attuale collocazione sconosciuta CRijksbureau voor Kunsthistoris che Documentatie.

la Madeleine di Jean Michel28. Tali componimenti erano proposti e rappresentati con tematiche ed episodi praticamente identici in varie lingue, come attestavano ad esempio la Conversione di Santa Maria Maddalena di Antonio di Jacopo Alamanni, o quella simile, ma di qualche anno più tarda in cui la santa dichiara: "I'sono ricca, nobile e pomposa, / Non mi curo ora di null' altra cosa"29. In questi testi, prima della sua conversione la santa suona spesso il liuto.

Tornando ai dipinti, per citarne solo alcuni si farà riferimento alle versioni di Amburgo ${ }^{30}$, Torino ${ }^{31}$, oppure in collezione privata ${ }^{32}$ (Fig. 3), in cui la santa, bellissima ed elegantissima, pizzica con grazia le corde del liuto. Non mancano le varianti in cui al posto del liuto si trova una tastiera, come in quella del Museo di Poznan ${ }^{33}$ (in cui sulle mensole alle spalle della santa compare uno strumento a fiato, forse un flauto dolce, come monito dei trascorsi poco meritevoli della santa) o quella di Jan Sanders van Hemessen del Worcester Art Museum ${ }^{34}$. È interessante notare come gli artisti, considerando le varie mani che si riuniscono sotto sotto la dicitura comune di Maestro delle Mezze Figure, avessero voluto inserire in alcune di queste scene anche allusioni al tema della vanitas, al trascorrere del tempo e alla perdita della bellezza. Alcune varianti mostrano infatti una candela spenta, oppure uno specchio: la candela, per citare alcune opere, si ritrova nella Maddalena che suona il liuto derivante dalla versione

28 ACCARIE, M. Le théâtre sacré de la fin du Moyen Âge: étude sur le sens moral de la Passion de Jean Michel. Ginevra: Librairie Droz, 1979.

29 D’ANCONA, A. editore. Sacre rappresentazioni dei secoli XIV, XV e XVI. Firenze: Successori Le Monnier, 1872, vol. 1, p. 266.

30 Olio su tavola, cm 37,5 x 26,8. Amburgo, Hamburger Kunsthalle, n. 76031528 (Maestro delle Mezze Figure).

31 Olio su tavola, cm 43 x 30. Torino, Galleria Sabauda, n. 4031528 (Maestro delle Mezze Figure).

32 Tra cui quella vista da Friedländer ad Hannover, nella collezione di Bernhard Hausmann (olio su tavola, cm 27.6 x 20.3. Il dipinto è stato recentemente venduto da Christie's, Important Old Master Pictures Londra, 10 luglio 2002, n. 16, Maesto delle Mezze Figure), oppure quella che lo studioso menzionava come nella collezione Klemperer di Dresda (olio su tavola, cm 37.2 x 25.2. Recentemente venduto da Sotheby's, Masters Paintings, New York, 22 maggio 2019, n. 23 (Maestro delle Mezze Figure).

33 Olio su tavola, cm 44 x 31. Poznań, Muzeum Narodowe w Poznaniu, n. Mo 115 (Maestro delle Mezze Figure).

34 Olio su tavola, cm 67,2 x 55,4. Worchester, Worcester Art Museum, n. 1920.88 (Jan van Hemessen) 
di Torino vista da Friedländer presso l'antiquario Hermann Ball di Berlino e venduta di Christie's nel $2009^{35}$ (Fig. 4), e in una che lo studioso faceva risalire alla casa d'aste Kleinberger ${ }^{36}$; mentre invece lo specchio si nota nella Maddalena che suona il clavicordo che nel 1929 si trovava a Berlino presso Paul Cassirer $^{37}$ (Fig. 5), variante del dipinto di Poznan. Le immagini che chi scrive ha potuto trovare di quest'ultimo esempio purtroppo non consentono di valutare con precisione l'immagine riflessa dallo specchio, ma sembra che si tratti della schiena della santa ${ }^{38}$. Un'Allegoria della caducità della bellezza di cui esistono varie copie, quasi tutte attribuibili all'ambito di Pieter Coecke, mostra una donna che suona il liuto, mentre un uomo regge davanti a lei uno specchio e alle sue spalleun teschio. Alcune varianti riportano l'iscrizione: "FORMOSAM SPECULO TE CERNES RESPICE FORMAM A TERGO POSITAM QUE NOTAT ESSE NICHIL" "39, non stupirebbe la possibilità di trovare una relazione tra questa massima e gli specchi delle sante, soprattutto tenendo conto che il modello della figura femminile dell'Allegoria a volte è lo stesso impiegato per la Maddalena Kleinberger. Queste allusioni alla transitorietà dei piaceri terreni insistono una volta di più sul comportamento negativo mantenuto dalla santa nel corso della sua vita.

Si è già accennato agli studi di Colim Slim, fondamentali per chi si interessi a queste opere, e va sottolineato come lo studioso non si fosse limitato ad approfondire l'aspetto musicale delle composizioni: oltre ad analizzarne le canzoni suonate aveva anche osservato come nel caso della Maddalena di Chatsworth House, la santa non stesse più suonando ma, nonostante avesse un'intavolatura aperta sul tavolo, avesse ormai messo via il liuto di cui nel dipinto si vede solo la custodia ${ }^{40}$. La Maddalena di Chatsworth House non è l'unica ad aver messo da parte il proprio strumento, ci sono anche una variante in cui la santa ha un liuto sulle ginocchia ma non suona ${ }^{41}$, e una in cui sta riponendo un clavicordo ${ }^{42}$. Si può quindi ipotizzare che ci siano alcune versioni che alludono ad un percorso di redenzione da parte di Maria Maddalena, preannunciato nell'uso di uno strumento a tastiera, nei simboli legati alla vanitas o in un elemento come la statuetta raffigurante Atena che si erge alla sommità del coperchio del vaso posto davanti alla finestra nella copia del dipinto di Torino. ${ }^{43}$

35 Olio su tavola, cm 54,7 x 42. Christie's, Old Master \& 19th Century Paintings, Drawings \& Watercolours Evening Sale, Londra, 8 Dicembre 2009, n. 2 (Maestro delle Mezze Figure).

36 Olio su tavola, cm 65 x 55. Vendita F. Kleinberger, New York, 18 novembre 1932, n. 34 (Nederlands Instituut voor Kunstgeschiedenis n. 31530, come: Anonimo neerlandese della prima metà del XVI secolo).

37 Olio su tavola, cm 27,5 x 20,5. Si veda: Nederlands Instituut voor Kunstgeschiedenis n. 31531 (Maestro delle Mezze Figure). Lo specchio si trova anche sullo sfondo di una versione in cui Maria Maddalena scrive una lettera venduta di recente dalla casa d'aste Aguttes e identificata con una "Giovane donna che scrive una lettera", olio su tela, cm 52 x 43, Hôtel des Ventes de Neuilly, 2-3 giugno 2014, n. 13 (Maestro delle Mezze Figure).

38 L'escamotage dello specchio era stato usato anche da Bellini in un altro contesto, si veda la Giovane donna nuda allo specchio di Vienna (olio su tavola, cm 62 x 79. Vienna, Kunsthistorisches Museum, n. 97, come Giovanni Bellini).

39 Olio su tavola, cm 38,6 x 29,5. Sotheby's, Old Master Paintings, Londra, 31 ottobre 2002, n. 28 (Cerchia di Pieter Coecke van Aelst).

40 COLIN SLIM, H. Mary Magdalene, Musician and Dancer. Early Music, 1980, Vol. 8, n 4, p. 460-473.

41 Olio su tavola, cm 102 x 73,5. Berlino, Gemäldegalerie, n. 59.4 (Jan Sanders van Hemessen).

42 Olio su tavola, cm 36,5 x 26. Collezione Mrs Clayton C. Timbrell, si veda: Nederlands Instituut voor Kunstgeschiedenis n. 31533 (Seguace del Maestro delle Mezze Figure).

43 Olio su tavola, cm 54,7 x 42. Christie's, Old Master \& 19th Century Paintings, Drawings \& Watercolours Evening Sale, Londra, 8 Dicembre 2009, n. 2 (Maestro delle Mezze Figure). 
Alla luce di queste osservazioni, si può dire che questa serie di dipinti raffiguranti Maria Maddalena che suona vadano letti piuttosto come allusioni all'aspetto meno morale del carattere di Maria Maddalena, e che di conseguenza l'elemento musicale stia ad indicare chiaramente un momento della vita della santa connesso alla sua condotta prima della conversione, quando si dedicava ancora ad un'esistenza mondana e frivola piuttosto che alla preghiera e all'ascetismo. Considerato il caso di Maria Maddalena, vale la pena confrontarlo con altri esempi, per verificare se quindi chiave di lettura valga a livello generale o se la si possa applicare solo alle opere appena viste.

Una categoria in cui le donne sono associate alla musica, e a vari strumenti musicali, è quella di personificazioni e allegorie. Le rappresentazioni delle stagioni, dei sensi, delle arti o delle Muse, spesso mostrano donne che suonano o che si trovano circondate da strumenti, come nel caso dell'Estate di Maarten de Vos incisa da Philps Galle, dove la figura principale suona il liuto, nel

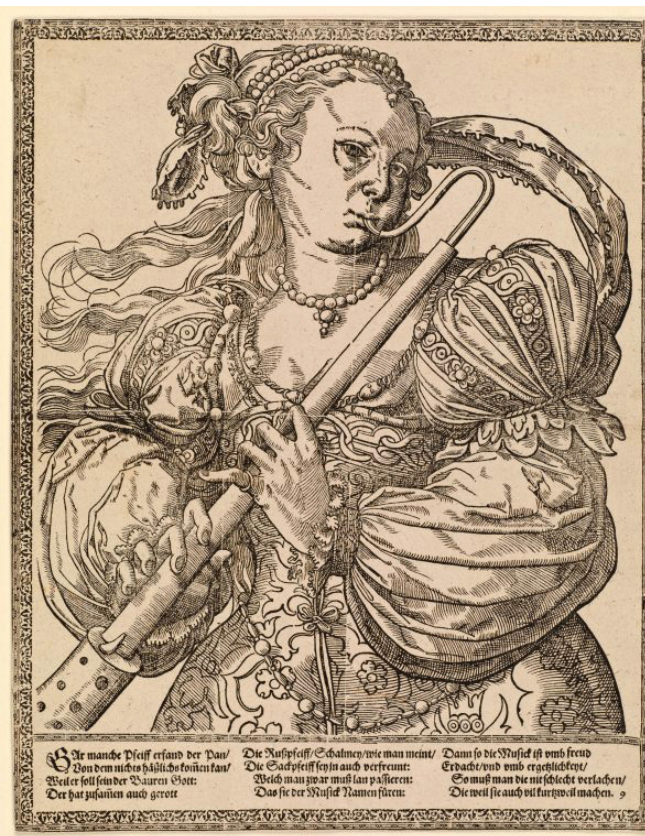

Fig. 6: Tobias Stimmer, Le Muse, New York, New York Public Library The Miriam and Ira D. Wallach Division of Art, Prints and Photographs: Print Collection, The New York Public Library. "The Bassoonist" New York Public Library Digital Collections. Accessed June 17, 2020. http://digitalcollections.nypl.org/items/ abbfI6c4-4c46-be4e-e040-e00aI 8065303 C New York Public Library.

Paesaggio con le nove Muse di Pozzoserrato venduto da

Sotheby's nel $2003^{44}$, o nelle più tarde Muse di Tobias Stimmer. Una serie completa ne è conservata alla New York Public Library ${ }^{45}$, si tratta di dieci fogli che raffigurano nove giovani e belle musiciste più una parodia, una vecchia che invece di suonare uno strumento sbatte un mestolo contro un piatto e una brocca. Nelle immagini compaiono vari tipi di strumenti, inclusi il liuto e i fiati. Il fatto che si trattasse di raffigurazioni delle Muse, e non di ritratti di donne reali, consentiva all'artista tedesco di raffigurarle intente a suonare anche strumenti che una dama non avrebbe mai toccato, come quelli a fiato (Fig. 6). Lo stesso valeva per altre serie con soggetti simili di altri maestri, come le personificazioni di arti e scienze di Etienne Delaune, in cui ogni figura era rappresentata in piedi, tra simboli allusivi della propria conoscenza, mentre suonava uno strumento, dal liuto, al triangolo, alla lira.

Le allegorie della Musica erano numerose, da quelle più retoriche come la composizione di Frans Floris incisa da Cornelis Cort - in cui però Musica suona un virginale mentre il liuto è lasciato ad un'altra figura femminile - alle varie immagini impiegate nei manuali di musica, come quella attribuita alla bottega di Lucas Cranach ed inserita nel trattato Practica Musica di Hermann Finck, in cui si vede una donna elegantemente vestita che suona il liuto circondata da altri strumenti. Nel caso di figure allegoriche e mitologiche si può tuttavia difficilmente trovare un riscontro con la vita reale e con il contesto dei ritratti dal vero: il loro carattere simbolico le faceva rientrare in un ambito in cui potevano essere concessi atteggiamenti e caratteristiche non altrimenti accettati.

44 Paesaggio con le nove Muse, olio su tela, cm 98,7 x 200,7. Sotheby’s, New York, 23 gennaio 2003, n. 56 (Lodewijk Toeput).

45 LA RUE, J., HOLLAND, J. B. Stimmer's women musicians: a unique series of woodcuts. Bulletin of the New York public library, 1960, vol. 64, n 1, p. 9-28. 


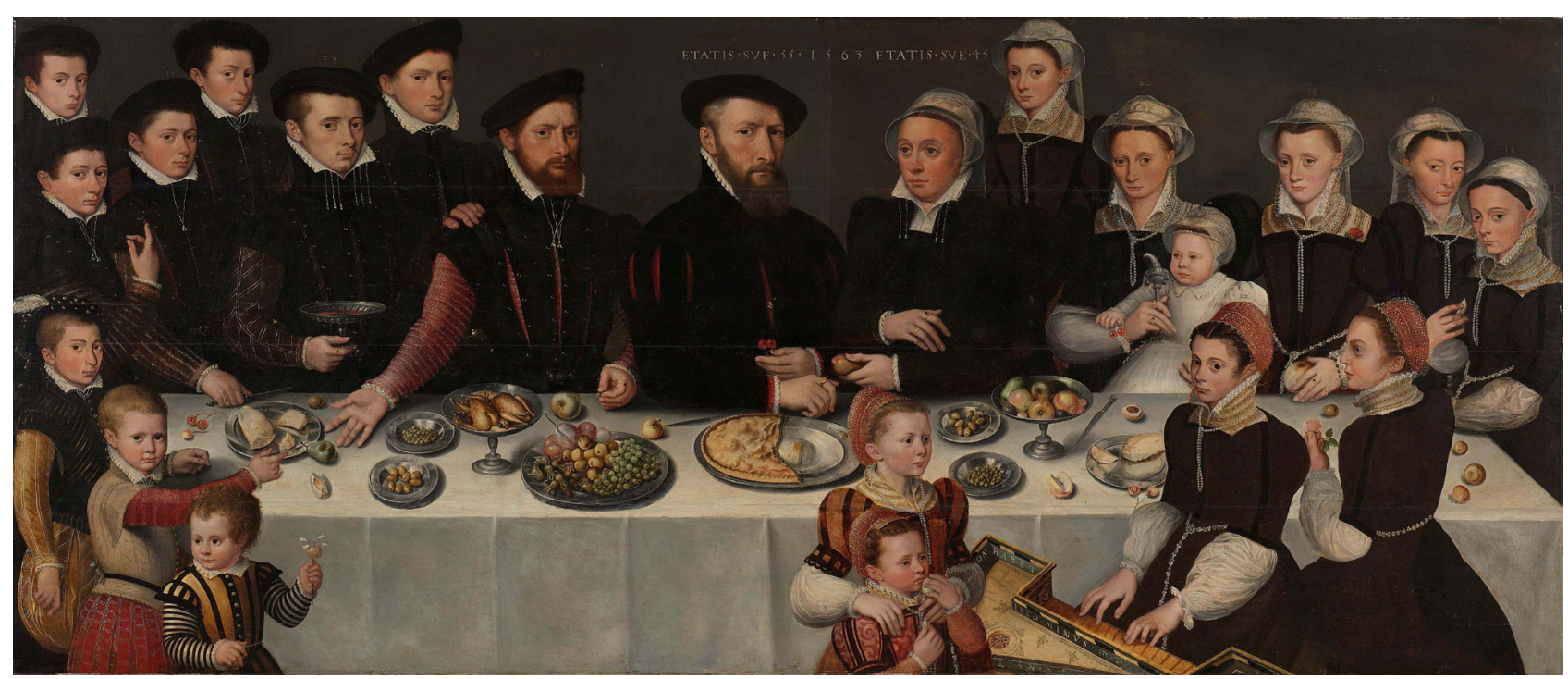

Fig 7: Anonimo, Ritratto di Pierre de Moucheron, Isabean de Gerbier e della loro famiglia, Amsterdam, Rijksmuseum (C) Rijksmuseum.

Il confronto più significativo ai fini di questo studio sarà pertanto con i ritratti, e una volta escluse le opere con chiaro significato allegorico o erotico, i ritratti femminili in cui l'effigiata, o una delle effigiate, suona non sono così numerosi. In primo luogo vanno menzionati i ritratti di famiglia. Soprattutto dopo la metà del XVI secolo, nell'ambito della tipologia del ritratto di gruppo che i Paesi Bassi apprezzavano particolarmente, si ritrovavano vari esempi in cui una rappresentante femminile della famiglia era raffigurata alla spinetta o al virginale. Si pensi al Ritratto di famiglia di Frans Floris conservato nello Stedelijk Museum Wuyts-Van Campen en Baron Caroly di Lier ${ }^{46}$, all'anonimo Ritratto di Pierre de Moucheron, Isabeau de Gerbier e della loro famiglia ${ }^{47}$ (Fig. 7), o al Ritratto di Hendrik van den Broucke e della sua famiglia attribuito a Cornelis de Zeeuw ${ }^{48}$, in ognuno di essi una delle figlie, con il volto serio e lo sguardo inespressivo, muove con grazia le dita sulla tastiera di strumenti elegantemente decorati ${ }^{49}$. Si tratta quindi di un contesto accettabile, l'intimità familiare, e di uno strumento considerato decoroso per un'esecuzione femminile, in accordo con la maggior parte dei manuali e dei trattati dell'epoca. La situazione in realtà non cambia quando si tratta di ritratti individuali. Di nuovo ci troviamo di fronte a giovani donne che suonano principalmente spinette, virginali e clavicordi. L'esempio forse più noto èdovuto a Catharina van Hemessen, la cui abilità di musicista era peraltro ben nota. L'artista ha rappresentato una donna - a volte identificata con la sorella, a volte con Catharina stessa - seduta davanti a un virginale ${ }^{50}$. Si è già ricordata la fanciulla che compone $\mathrm{o}$ trascrive una melodia venduta da Kollenburg, e Popham inseriva nel catalogo delle incisioni di Jan Cornelisz Vermeyen una Donna al clavicordo di cui una copia è conservata al British Museum ${ }^{51}$. Van-

46 Olio su tavola, cm 130 x 225. Lier, StedelijkMuseum Wuyts-Van Campen en Baron Caroly, n. 52 (Frans Floris).

47 Olio su tavola, cm 108 x 246. Amsterdam, Rijksmuseum, n. SK-A-1537 (Maestro del Ritratto della famiglia de Moucheron).

48 Olio su tavola, cm 146 x 220. Münster, LWL - Museum für Kunst und Kultur, n. 1348 LM (Cornelis de Zeeuw).

49 Per un'analisi degli strumenti riprodotti in questi dipinti si veda: RIPIN, E. M. Joest Karest's Virginal and the origins of the Flemish tradition. In: Edwin M. RIPIN, editore. Keyboard Instruments. Studies in Keyboard Organology, 1500-1800. Edimburgo: Edinburgh University Press, 1971, p. 67-75.

50 Olio su tavola, cm 32,2 x 25,7. Colonia, Wallraf-Richartz-Museum, n. WRM 654 (Catharina van Hemessen).

51 POPHAM, A. E. Catalogue of Etchings by Jan Cornelisz Vermeyen. Oud Holland, 1927, n 44, p. 174-182, p. 180, n. 20. Si veda anche un disegno (inchiostro bruno e bistro su carta, cm 27.8 x 20.7) conservato a Berlino, Kupferstichkabinett, n. KdZ 516 (Jan Cor- 
no qui ricordate anche le Maddalene di van Hemessen, alcune delle quali potrebbero non essere solo immagini della santa. Una è la versione di Berlino in cui la figura principale tiene il liuto posato sulle ginocchia, il volto meno stereotipato delle altre varianti e gli abiti lascerebbero pensare ad una caratterizzazione maggiore. Ancora nell'ambito della produzione legata a van Hemessen, Burr Wallen ${ }^{52}$ identificava la già citata Maddalena al clavicordo di Worchester ${ }^{53}$ con Margherita di Parma, la figlia naturale di Carlo V cresciuta a Bruxelles ma sotto la tutela di Margherita d'Asburgo. Lo studioso non spiegava perché la giovane Margherita sarebbe stata ritratta come Maria Maddalena, ma tale scelta, se davvero l'identificazione fosse corretta, potrebbe essere motivata dal forte legame che stringeva Margherita d'Asburgo e i suoi antenati Borgognoni alla santa ${ }^{54}$. La governatrice dei Paesi Bassi si era fatta a sua volta ritrarre con gli attributi di santa Maddalena e non stupirebbe che avesse voluto che anche la sua protetta crescesse seguendo una devozione tradizionale per la famiglia.

Un ritratto leggermente più tardo in cui si riconosce Maria van Woerden van Vliet mostra l'effigiata in un interno, alle sue spalle si vede uno strumento a corde, forse un liuto, e un quaderno di musica ${ }^{55}$. In questo caso non ci sarebbero solo la solitudine e l'intimità dell'ambiente domestico a proteggere la reputazione della donna - che ad ogni modo non sta suonando, evitando quindi comunque di mostrarsi al pubblico in un atteggiamento poco conveniente - ma anche il fiore che tiene in mano. L'immagine della vendita non consente di distinguere se si tratti di un garofano bianco simbolo del matrimonio, o di una rosa bianca, simbolo dell'amore spirituale, ma è piuttosto evidente che si tratti di un ritratto di fidanzamento. C'è tuttavia un altro ritratto individuale in cui si vede una donna che suona il liuto senza avere l'aspetto di una cortigiana, si tratta di un dipinto attribuito al Maestro degli anni '40 e conservato a Parigi, presso la Fondazione Custodia ${ }^{56}$. Mentre l'effigiata suona, tiene sulla spalla un pappagallo, che, generalmente associato a Maria e all'annunciazione, era simbolo di purezza, innocenza e a volte anche prefigurazione del matrimonio. La sua presenza potrebbe quindi garantire alla donna una dimensione socialmente accettabile, potrebbe, anche in questo caso, trattarsi di un ritratto nuziale.

Le caratteristiche dei ritratti individuali rispecchiano pertanto una volta di più $i$ testi formativi dell'epoca, e si mostrano coerenti con le raffigurazioni di Santa Maddalena per quanto riguarda gli strumenti e le esecuzioni musicali: contrariamente alla santa, le giovani di buona famiglia vengono rappresentate solo alla tastiera mentre eseguono musica di intrattenimento - un intrattenimento decente e decoroso - per la propria austera famiglia.

Infine, a ribadire l'importanza del contesto e del tipo di strumenti, vanno menzionate ancora alcune serie di pochi elementi che raffigurano suonatrici vestite in maniera ricercata e senza alcuna connotazione individuale o ambientale. Come le serie di Tobias Stimmer e Etienne Delaune, alcuni fogli

nelisz. Vermeyen).

52 WALLEN, B. Jan Sanders Van Hemessen: An Antwerp Painter Between Reform and Counter-Reform. Ann Arbour, MI: UMI Research Press, 1983, p. 12; 32.

53 Olio su tavola, cm 67,2 x 55,4. Worchester, Worchester Art Museum, n. 1920.88 (Jan van Hemessen).

54 Si veda: BELLAVITIS, M. Tra ritratto e devozione, immagini della Maddalena nella prima metà del Cinquecento, Critica d'Arte.2013 [2015], LXXV, ${ }^{\circ}$ 55-56, p. 19-36, con bibliografia di riferimento.

55 Ritratto di Maria van Woerden van Vliet (1540-1603), olio su tavola, cm 82 x 56. Dorotheum, Vienna, 18 ottobre 1994 , n. 69 (Aert o Pieter Pietersz. I).

56 Olio su tavola, cm 70 x 55. Parigi, Fondation Custodia - Collection Frits Lugt, n. 4994 (Maestro degli Anni ‘40). 
sono stati prodotti in altri contesti geografici, ma il legame con l'ambito neerlandese è comunque innegabile, e fanno pensare ai concerti campestri e alle rappresentazioni del figliol prodigo tra le cortigiane che erano tanto in voga attorno alla metà del XVI secolo. Alcuni fogli riconducibili ad uno stesso gruppo, ma forse appartenenti ad edizioni differenti, si trovano al British $\mathrm{Mu}-$ seum e al Metropolitan Museum of Art. Vi sono raffigurate una suonatrice di dulcimer, a Londra, mentre gli strumenti che compaiono nelle silografie di New York sono un organo portativo (Fig. 8) e un flauto traverso. I fogli sono ritagliati e non ci sono riferimenti al loro contesto originale, le similitudini con le Muse di Tobias Stimmer sono evidenti ma sembrerebbe che non sia data qui la stessa importanza all'elemento allegorico. Alla Bibliothèque Nationale di Parigi è conservato un concerto in cinque fogli più o meno coevo, in ogni foglio compare una figura che suona di cui quattro

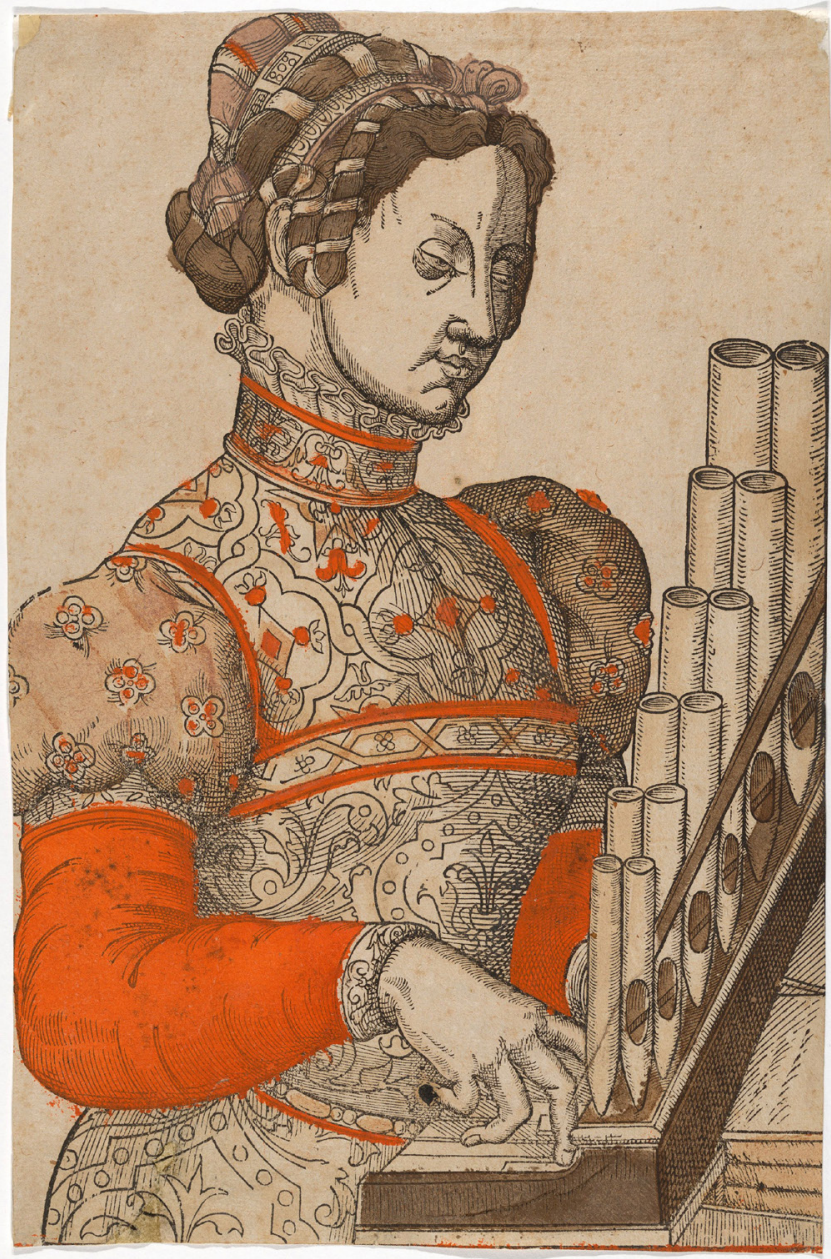

Fig. 8: Anonimo francese, XVI secolo, Donna che suona un organo portativo, New York, Metropolitan Museum, n. 20I3.942 C Metropolitan Museum of Art. sono donne, mentre la quinta è un uomo che suona il cornetto e dirige l'insieme ${ }^{57}$. La suonatrice di organo proviene dallo stesso modello di quella del Metropolitan Museum, mentre le altre si discostano sia dalle Muse di Stimmer che dai fogli di Londra e New York. Il carattere erotico di queste serie è evidenziato dai versi che accompagnano il direttore delle musiciste di Pariigi, la cui conclusione è generalmente intesa come un doppio senso:

Mes Dames chacune de vous,

Entende à tenire sa partie,

Nostre Concert sera plus doux,

La Musique estant assortie.

Serves vous de vos instrumentz,

Selon l'art gardez la mesure,

Faictes tous vos fredonnementz,

Non par B mol mais par nature.

57 Silografia, cm 500 x 370. Bibliothèque Nationale, n. Ea 17 réserve, t.2 (Anonimo francese, 1570 ca.). 
Considerando le testimonianze dell'epoca e confrontando gli esempi di rappresentazioni di donne che suonano uno strumento si può quindi dedurre che tra la fine del XV e la metà del XVI secolo una stretta familiarità con la musica fosse generalmente incoraggiata, ma con dei limiti precisi. Tali limiti riguardavano sia il tipo di musica che il tipo di strumento la cui pratica era concessa ad una donna per bene. Il fatto che, escludendo il caso delle figure allegoriche, gli strumenti raffigurati e la musica eseguita variassero a seconda del contesto, della situazione e del soggetto raffigurato fa pensare che si trattasse di convenzioni generalmente acquisite e molto chiare per la cultura e la morale dei Paesi Bassi dell'epoca. Questo non vuol dire che, ad esempio, il liuto fosse uno strumento proibito, al contrario, i documenti dimostrano come fosse molto comune anche tra le fanciulle. Tuttavia, le norme di comportamento che regolavano il rapporto delle future dame con la società imponevano che venisse osservata una certa etichetta musicale. Allo stesso modo, il repertorio canoro delle giovani non si limitava di sicuro ai salmi e ai canti devozionali proposti per imparare il catechismo. Danze e canzoni d'amore erano sicuramente ben note a tutte, soprattutto verso la fine del secolo, come provano il manoscritto di Susanne von Soldt, una raccolta di salmi e danze franco-fiamminghe posseduto dalla giovane figlia di un mercante anversese trasferitosi a Londra ${ }^{58}$, e la dedica di Jean de Castri a Marguerite e Beatrice Hooftamns:

Vertueuses et discrettes jeunes damoyselles, Marguerite et Beatrice Hooftmans seurs germains ... qui sont Chansons, Stanses, Sonets, Epigrammes à deux parties tant seulement par moy mis en Musique, lesquelles m'ont semblé bien propres \& convenables vous presenter, pour avec que icelles à la fois recréer voz Esprits lassé \& recreuz de voz affaires privées \& domestiques vous priant tantost prenant de Luth, tantost l'Espinette en voz blanches polies \& délicates mains, deigner marier voz doucettes voiz à l'harmonie d'icelles, ce que causera \&à bon droit qu'on vous appeller Marguerite \& Beatrice ... en la Musicque les non pareilles ... De voz bonnes graces humble serviteur, Jean de Castro ${ }^{59}$.

58 CURTIS, A., editore. Monumenta Musica Neerlandica: Dutch Keyboard Music of the 16th and 17th Centuries. Utrecht: Vereniging voor Nederlandse Muziekgeschiedenis, 1961; FORNEY, K. K. A Proper Musical Education for Antwerps Women. In: S. FORSCHER WEISS, R. E. MURRAY Jr., C. J. CYRUS, editori. Music Education in the Middle Ages and the Renaissance Indiana. Bloomington, IN: Indiana University Press, 2010, p. 84-125, p. 106.

59 DE CASTRO, J. Chansons, stanses, sonets et épigrammes à deux parties. Anversa: Phalèse et Bellère, 1592. 


\section{Bibliografia}

ACCARIE, Maurice. Le théâtre sacré de la fin du Moyen Âge: étude sur le sens moral de la Passion de Jean Michel. Ginevra: Librairie Droz, 1979.

ATTAINGNANT, Pierre. Premier livre contenant XXXI chansons musicales, Paris, 1535.

BELLAVITIS, Maddalena. Tra ritratto e devozione, immagini della Maddalena nella prima metà del Cinquecento, Critica d'Arte.2013 [2015], LXXV, n 55-56, p. 19-36. ISSN 0011-1511.

BLACKBURN, Bonnie J. Anna Inglese and Other Women Singers in the Fifteenth Century: Gleanings from the Sforza Archives. In: K. Forney e J.L. Smith, editori. Sleuthing the Muse: Essays in Honor of William F. Prizer, Festschrift Series $n^{\circ}$ 26. Hillsdale, NY: Pendragon Press, 2012, p. 237-52. ISBN 1576471489.

BORROFF, Edith. Women Composers: Reminiscence and History. College Music Symposium. 1975, vol. 15, p. $26-33$. ISBN 00695696.

BOWERS, Jane M., TICK, Judith, editori. Women Making Music: The Western Art Tradition, 1150-1950. Urbana e Chicago: University of Illinois Press, 1987. ISBN 9780252014703.

BRUTO, Giovanni Michele. La institutione di una fanciulla nata nobilmente. Anversa: Jehan Bellére, 1555.

BRYCE, Judith. Performing for Strangers: Women, Dance, and Music in Quattrocento Florence. Renaissance Quarterly, 2001, vol. 54, $n^{\circ}$ 4, Part 1, p. 1074-1107, ISSN 0034-4338.

CAGNOLATI, Antonella. Giovanni Michele Bruto e l'educazione femminile: La Institutione di una fanciulla nata nobilmente (1555). Annali dell'Univeristà di Ferrara, Nuova serie, Filosofia, Discussion Papers. 2001,n 64, p. 1-22. ISSN 1824-3770.

CASTIGLIONE, Baldassare. Il cortegiano. Venezia: Eredi di Aldo Manuzio, 1545.

COMELLAS SOLÉ, Jordi. La música dentro de las cortes europeas del siglo XVI. El modelo de Il Cortegiano y el papel de las damas en su consolidación. El ejemplo de las cortes italianas. Philostrato. Revista de Historia y Arte. 2020, n 7, p. 5-32 [consultato: 3 luglio 2020]. ISSN 2530-9420. Disponibile alla pagina: https://doi.org/10.25293/philostrato.2020.01.

COLIN SLIM, Harry. Mary Magdalene, Musician and Dancer. Early Music, 1980, Vol. 8, n 4, p. 460-473. ISSN 03061078 .

COLIN SLIM, Harry. Paintings of Lady Concerts and the Transmission of "Jouissance vous donneray". Imago Musicae, 1984, nº I, p. 51-73. ISBN 3761807198.

COLtON, Lisa, HAWORTH, Catherine, editori. Gender, Age and Musical Creativity. Farnham: Ashgate Publishing, 2015. ISBN 9781472430854. 
CURTIS, Alan, editore. Monumenta Musica Neerlandica: Dutch Keyboard Music of the 16th and 17th Centuries. Utrecht: Vereniging voor Nederlandse Muziekgeschiedenis, 1961.

D’ANCONA, Alessandro, editore. Sacre rappresentazioni dei secoli XIV, XV e XVI. Firenze: Successori Le Monnier, 1872.

DE BOURDEILLE DE BRANTOME, Pierre. Vies des dames galantes. Edizione Frères Garnier, Parigi, 1841.

DE CASTRO, Jean. Chansons, stanses, sonets et épigrammes à deux parties. Anversa: Phalèse et Bellère, 1592.

DE MIRIMONDE, Albert P. La musique chez les peintres de la fin de l'ancienne école de Brouges. Jaarboek van het Koninklijk Museum voor Schone Kunsten-Antwerpen. 1976, p. 25-81. ISSN 0770-3104.

DENNIS, Flora. Unlocking the Gates of Chastity Music and the Erotic in the Domestic Sphere in Fifteenth-and Sixteenth-Century Italy. In: Sara F. MATTHEWS-GRIECO, editore. Erotic cultures of Renaissance Italy. Visual Culture in Early Modernity. Aldershot: Ashgate, 2010, p. 223-246. ISBN 0754662144.

DUGGAN, Mary K. Queen Joanna and Her Musicians. Musica Disciplina, 1976, vol. 30, p. 73-95. ISSN 0077-2461.

ERASMO, Desiderio. Christiani matrimonii institutio, Apud Inclytam Basileam, 1526.

FORNEY, Kristine K. Nymphes gayes en abry du Laurier: Music Instruction for the Bourgeois Woman. Musica Disciplina, 1995, vol. 49, p. 151-187, ISSN 0077-2461.

FORNEY, Kristine K. A Proper Musical Education for Antwerps Women. In: Susan FORSCHER WEISS, Russell E. MURRAY Jr., Cynthia J. CYRUS, editori. Music Education in the Middle Ages and the Renaissance Indiana. Bloomington: Indiana University Press, 2010, p. 84-125. ISBN 0253354862.

FRIEDLÄNDER, Max J. Early Netherlandish Paintings. XII. Jan van Scorel and Pieter Coeck van Aelst. Edizione a cura di Henri PAUWELS e Geert LEMMENS. Leida: Sijthoff, e Bruxelles: La Connaissance, 1975. ISBN 9028601953.

FRIJHOFF, Willem, e SPIES, Marijke. Dutch Culture in a European Perspective: 1650, Hard-Won Unity. Assen: Van Gorcum, 2004. ISBN 1403932271.

GOSS THOMPSON, Glenda. Mary of Hungary and Music Patronage. The Sixteenth Century Journal, 1984a, vol. 15, $\mathrm{n}^{\circ}$ 4, p. 401-418. ISSN 0361-0160.

GOSS THOMPSON, Glenda. Music in the Court Records of Mary of Hungary, Tijdschrift van de Vereniging voor Nederlandse Muziekgeschiedenis, 1984b, vol. 34, n² 2, p. 132-173. ISSN 1869-1874.

GUICCIARDINI, Lodovico. Descrittione Di M. Lodovico Gvicciardini patritio fiorentino, di tutti i Paesi Bassi, altrimenti detti Germania Inferiore: Con più carte di Geographia del paese, \& col ritratto naturale di più terre principali. Anversa: Guglielmo Silvio, 1567.

HARASZTI, Emile. Marie de Hongrie et son Ungarescha. Revue de Musicologie, 1930, vol. 11, $n^{\circ}$ 35, p. 176-194. ISSN $0035-1601$. 
HEARTZ, Daniel. Mary Magdalen, Lutenist. Journal of the Lute Society of America, 1972, n 5, p. 52-67. ISSN 0076-1526.

JARDINE, Lisa. 'O Decus Italiae Virgo', or The Myth of the Learned Lady in the Renaissance. The Historical Journal, 1985, vol. 28, n 4, p. 799-819. ISSN 0018-246X.

LA RUE, Jan, HOLLAND, Jeanette B. Stimmer's women musicians: a unique series of woodcuts. Bulletin of the New York public library, 1960, vol. 64, n 1, p. 9-28. ISSN0028-7466.

LAMARY, Thomasin. Musical Voices of Early Modern Women: Many-Headed Melodies. Londra: Routledge, 2017. ISBN 1138258776.

LORENZETTI, Stefano. "Quel celeste cantar che mi disface”. Immagini della donna ed educazione alla musica nell'ideale pedagogico del Rinascimento italiano. Studi Musicali, 1994, Anno XXIII, n 2, p. 241-262. ISSN $2037-6413$.

LORENZETTI, Stefano. Public behavior, music and the construction of feminine identity in the Italian Renaissance. Recercare, 2011, vol. 23, n 1-2, p. 7-34. ISSN 1120-5741.

MAURIER, Gabriel. La guirlande des Jeunes filles, bastie et composee en langue francoise et flamengue, et de nouveau reveue et translatee de francois en haut alleman par Abraham des Mans. Edizione: Colonia, 1597.

MECONI, Honey. Pierre de la Rue and Musical Life at the Habsburg-Burgundian Court. Oxford: Oxford University Press, 2003. ISBN 9780198165545.

MOENS-HAENEN, Greta. Music at the court of Marguerite of Austria. Jaarboek van het Vlaams Centrum voor Oude Muziek. 1987. ISBN 9789068530193.

PARKINSON, John A. A Chanson by Claudin de Sermisy. Music \& Letters, 1958, vol. 39, № 2, p. 118-122. ISSN 0027-4224.

PICKER, Martin. The Chanson Albums of Marguerite of Austria: Manuscripts 228 and 11239 of the Bibliothèque Royale de Belgique, Bruxelles. Berkeley: University of California Press, 1960.

POPHAM, Arthur E. Catalogue of Etchings by Jan Cornelisz Vermeyen. Oud Holland, 1927, n 44, p. 174-182. ISSN 0030-672X.

PRIZER, William F. Isabella d'Este and Lorenzo Da Pavia, 'Master Instrument-Maker'. Early Music History, 1982, 2, p. 87-127. ISSN 0261-1279.

PRIZER, William F. Isabella d'Este and Lucrezia Borgia as Patrons of Music: The Frottola at Mantua and Ferrara. Journal of the American Musicological Society, 1985, vol. 38, n 1, p. 1-33. ISSN 0003-0139.

PRIZER, William F. Games of Venus: Secular Vocal Music in the Late Quattrocento and Early Cinquecento. The Journal of Musicology, vol. 9, $\mathrm{n}^{\circ}$ 1, 1991, p. 3-56, ISSN 15338347.

PRIZER, William F. Una “Virtù Molto Conveniente A Madonne”: Isabella D’este as a Musician. The Journal of Musicology, 1999, vol. 17, n 1, A Birthday Tableau for H. Colin Slim, p. 10-49. ISSN 15338347. 
PRIZER, William F. Renaissance Women as Patrons of Music: The North-Italian Courts. In: Thomas SCHMIDT-BESTE, editore. Institutions and Patronage in Renaissance Music. Aldershot: Ashgate, 2012, p. 161-190. ISBN 0754629325.

RIPIN, Edwin M. Joest Karest's Virginal and the origins of the Flemish tradition. In: Edwin M. RIPIN, editore. Keyboard Instruments. Studies in Keyboard Organology, 1500-1800. Edimburgo: Edinburgh University Press, 1971, p. 67-75. ISBN 0486233634.

SCHIESARI, Juliana. In Praise of Virtuous Women? For a Genealogy of Gender Morals in Renaissance Italy. Annali d'Italianistica, 1989, vol. 7, Women's Voices in Italian Literature, p. 66-87. ISSN 0741-7527.

SHEPHARD, Tim. Constructing Isabella d'Este's musical decorum in the visual sphere, Renaissance Studies, 2011, vol. 25, n 5, p. 684-706. ISSN 02691213.

SHEPHARD, Tim. Echoing Helicon: Music, Art and Identity in the Este Studioli, 1440-1530. Oxford: Oxford University Press, 2014. ISBN 0199936137.

SCHNEIDER, Federico. An Amused Muse: The donna di palazzo and Music in the Cortegiano, L'analisi linguistica e letteraria, 2002, vol. 10, $\mathrm{n}^{\circ}$ 1-2, p. 449-460. ISSN 1827-7985.

STEBLIN, Rita. The Gender Stereotyping of Musical Instruments in the Western Tradition. Canadian University Music Review / Revue de musique des universités canadiennes, Voices of Women: Essays in Honour of Violet Archer Voix de femmes. Mélanges offerts à Violet Archer, 1995, vol. 16, n 1, p. 128-144. ISSN 0710-0353.

STRAS, Laurie. Women and Music in Sixteenth-Century Ferrara (New Perspectives in Music History and Criticism). Cambridge: Cambridge University Press, 2018. ISBN 1107154073.

TINCTORIS, Johannes. Complexus effectuum musices, 1472-75 [consultato: 26 giugno 2020]. Disponibile alla pagina http://www.chmtl.indiana.edu/tml/15th/TINCOM1 TEXT.html.

VALERY, Magdaleine. La Montaigne des pucelles. Den maeghdenbergh. Leiden: Jan Paets, 1599.

VAN PELT, Nadia T. Drama in Medieval and Early Modern Europe: Playmakers and their Strategies. Londra: Routledge, 2019. ISBN 1138189375.

VECCE, Carlo. Modelli della pedagogia umanistica dall'Italia all'Europa. In: Maria Da Rif, editore. Civiltà italiana e geografie d'Europa. XIX Congresso AISLLI 19-24 settembre 2006, Trieste - Capodistria - Padova - Pola. Trieste: EUT Edizioni Università di Trieste, 2009, p. 146-154. ISBN 9788883032622.

WALLEN, Burr. Jan Sanders Van Hemessen: An Antwerp Painter Between Reform and Counter-Reform. Ann Arbour, MI: UMI Research Press, 1983. ISBN 0835715175.

ZECHER, Carla. The Gendering of the Lute in Sixteenth-Century French Love Poetry. Renaissance Quarterly, 2000, vol. 53, n 3, p. 769-791. ISSN 0034-4338. 